\title{
Complex gamma-ray behavior of the radio galaxy M 87
}

\author{
Faical Ait Benkhali ${ }^{1}$, Nachiketa Chakraborty ${ }^{1}$, and Frank M. Rieger ${ }^{1,2}$ \\ 1 Max-Planck-Institut für Kernphysik, Saupfercheckweg 1, 69117 Heidelberg, Germany \\ e-mail: F.Ait-Benkhali@mpi-hd.mpg.de, cnachi@mpi-hd.mpg.de, frank.rieger@mpi-hd.mpg.de \\ 2 ZAH, Institut für Theoretische Astrophysik, Universität Heidelberg, Philosophenweg 12, 69120 Heidelberg, Germany
}

Received 21 November 2017 / Accepted 6 August 2018

\begin{abstract}
Context. In recent years, non-blazar active galactic nuclei (AGN) such as radio galaxies have emerged as a highly instructive source class providing unique insights into high energy acceleration and radiation mechanisms.

Aims. Here we aim to produce a detailed characterization of the high-energy (HE; >100 MeV) gamma-ray emission from the prominent radio galaxy M 87.

Methods. We analyzed approximately eight years of Fermi-LAT data and derived the spectral energy distribution between $100 \mathrm{MeV}$ and $300 \mathrm{GeV}$. We extracted lightcurves and investigated the variability behavior for the entire energy range as well as below and above $10 \mathrm{GeV}$

Results. Our analysis provides (i) evidence for HE gamma-ray flux variability and (ii) indications for a possible excess over the standard power-law model above $E_{\mathrm{b}} \sim 10 \mathrm{GeV}$, similar to the earlier indications in the case of Cen A. When viewed in HE-VHE context, this is most naturally explained by an additional component dominating the highest-energy part of the spectrum. Investigation of the $\gamma$-ray lightcurves suggests that the lower-energy $(<10 \mathrm{GeV})$ component is variable on timescales of (at least) a few months. The statistics of the high energy component $(>10 \mathrm{GeV})$ does not allow significant constraints on variability. We do, however, find indications for spectral changes with time that support variability of the putative additional component and seem to favor jet-related scenarios for its origin capable of accommodating month-type variability.

Conclusions. The current findings suggest that both the high-energy $\left(>E_{\mathrm{b}}\right)$ and the very high energy (VHE; $\left.>100 \mathrm{GeV}\right)$ emission in M 87 are compatible with originating from the same physical component. The variability behavior at VHE then allows further constraints on the location and the nature of the second component. In particular, these considerations suggest that the VHE emission during the quiescent state originates in a similar region as during the flare.
\end{abstract}

Key words. radiation mechanisms: non-thermal - galaxies: active - gamma rays: galaxies - galaxies: jets galaxies: elliptical and lenticular, cD - galaxies: individual: M 87

\section{Introduction}

The giant elliptical Virgo cluster galaxy M 87 (NGC 4486, 3C274) is known for its prominent one-sided jet extending up to kiloparsec scales and for its extraordinary nuclear activity manifesting itself across all wavebands up to $\mathrm{TeV}$ energies (e.g., Marshall et al. 2002; Kovalev et al. 2007; Rieger \& Aharonian 2012). Being the second nearest (distance $d \simeq 16.4 \pm 0.5$, Bird et al. 2010) active galaxy, M 87 has been commonly classified as a low-excitation, weak-power Fanaroff-Riley (FR) I radio galaxy with a jet considered to be misaligned by $i \sim\left(15^{\circ}-25^{\circ}\right)$ (e.g., Acciari et al. 2009). Despite of hosting a very massive black hole $(\mathrm{BH})$ in the range of $M_{\mathrm{BH}} \simeq(2-6) \times 10^{9} M_{\odot}$ (Marconi et al. 1997; Gebhardt \& Thomas 2009; Walsh et al. 2013), M 87 has been found to be highly underluminous suggesting that accretion onto its $\mathrm{BH}$ proceeds in a radiatively inefficient mode (e.g., Reynolds et al. 1996). Given its proximity and large gravitational reference scale, $r_{g}=G M_{\mathrm{BH}} / c^{2}=6 \times 10^{14}\left(4 \times 10^{9} M_{\odot} / M_{\mathrm{BH}}\right) \mathrm{cm}$, M 87 has been the focus of numerous observational campaigns enabling to study astrophysical processes such as for example, jet formation or the production of non-thermal radiation in exceptional detail (Doeleman et al. 2012; Rieger \& Aharonian 2012; Kuo et al. 2014; Hada et al. 2014, 2016; Mertens et al. 2016).

M 87 was the first extragalactic source detected at very high energies (VHE; $>100 \mathrm{GeV}$ ). It is well known for showing rapid (day-scale) VHE variability during active states and a hard, featureless spectrum (power-law photon index $\Gamma=2.2 \pm 0.2$ in high, and somewhat steeper $\Gamma \sim 2.6$ in low states) extending up 10 TeV. (Aharonian et al. 2003, 2006; Albert et al. 2008; Acciari et al. 2009; Aliu et al. 2012; Abramowski et al. 2012). At high energies (HE; $>100 \mathrm{MeV}$ ), Fermi-LAT has reported the detection of gamma-ray emission from M 87 up to $30 \mathrm{GeV}$ based on the first 10 months of LAT data (Abdo et al. 2009), with a photon spectrum then compatible with a single power-law of index $\Gamma=2.26 \pm 0.13$, that is, similar to the one(s) in the VHE high states. Despite these similarities, a simple extrapolation of this HE power-law to the VHE regime turns out to be insufficient to account for the flux levels measured during the $\mathrm{TeV}$ high states (Rieger \& Aharonian 2012). The HE lightcurve (on $10 \mathrm{~d}$ bins) for these early observations does not show evidence for significant flux variations, though the occurrence of shortertimescale variations cannot be excluded. The updated third FermiLAT Point Source Catalog (3FGL) based on four years of Pass 7 reprocessed data gives similar results with the spectrum below $10 \mathrm{GeV}$ compatible with a single power-law of index $\Gamma=2.04 \pm$ 0.07 (Acero et al. 2015), yet suggesting a possible turnover above $10 \mathrm{GeV}$. The degree to which the TeV flux levels can be matched is thus an open issue. Our current work seeks to fill this gap using extended data sets. It is partly motivated by recent findings of an uncommon HE spectral hardening in the related source Cen A (Sahakyan et al. 2013; Brown et al. 2017).

A model comparison of the spectral energy distribution (SED) in M 87 indicates that a one-zone synchrotron selfCompton (SSC) approach, in which the non-thermal emission 
processes in radio galaxies are taken to be similar to that in $\mathrm{BL}$ Lacs but with modest Doppler boosting (Chiaberge et al. 2001) (misaligned BL Lac type with Doppler factor $D=1 /\left[\gamma_{\mathrm{b}}(1-\right.$ $\beta \cos i)]<4$ for M 87, where $\gamma_{\mathrm{b}}$ is the bulk flow Lorentz factor), is unable to account for its overall SED up to TeV energies (including the high, and evidently also, the low states), yet capable of reproducing the emission below $\sim 10 \mathrm{GeV}$ (e.g., Abdo et al. 2009). This suggests that part of the HE-VHE emission may originate from a different (additional) region. The analysis presented here casts new light on this.

In Sects. 2 and 3 we describe the results of a general HE spectrum and lightcurve analysis of approximately eight years of Fermi-LAT data, while Sect. 4 explores the evidence for different spectral states. The results are discussed in Sect. 5.

\section{Observations and analysis}

Fermi-LAT is a $e^{ \pm}$pair-conversion $\gamma$-ray detector sensitive to photons in the energy range from $\sim 20 \mathrm{MeV}$ to more than $300 \mathrm{GeV}$ (Atwood et al. 2009). It operates primarily in survey mode and continuously scans the whole sky every three hours.

For our current analysis we used PAss8 (P8R2) algorithms (Atwood et al. 2013) and data covering about 7.7 years from August 4, 2008 to April 12, 2016. A binned maximum likelihood analysis (Mattox et al. 1996) with a bin size of $0.1^{\circ}$ was performed using the standard Fermi Science Tools ${ }^{1}$ v10r0p5 software package. Events were selected with energies between $100 \mathrm{MeV}$ and $300 \mathrm{GeV}$ and in a region of interest (ROI) of $20^{\circ}$ around the position of M $87(\mathrm{RA}=187.705$, Dec = 12.3911) with P8R2_SOURCE_V6 instrument response functions (IRF). We performed standard quality cuts in accordance with PAss8 data analysis criteria. The background emission was modeled using the Galactic and isotropic diffuse emission gll_iem_v06 and iso_P8R2_SOURCE_V6_v06 files ${ }^{2}$. All sources from the third LAT source catalog (3FGL; Acero et al. 2015) within the ROI are included in the model to ensure a satisfactory background modeling. Specifically, for the bright sources 3FGL J1224.9+2122, 3FGL J1229.1+0202 and 3FGL J1239.5+0443 in the ROI, the spectral indices were allowed to vary in addition to varying the normalisations as for the other sources. Additionally photons coming from zenith angles larger than $90^{\circ}$ were all rejected to reduce the background from $\gamma$-rays produced in the atmosphere of the Earth (albedo). The test statistic value (TS), defined as $\mathrm{TS}=-2 \ln \left(L_{0} / L_{1}\right)$ was then used to determine the source detection significance, with threshold set to TS $=25(\sim 5 \sigma)$.

Our likelihood analysis reveals a point source with a high statistical significance $\mathrm{TS} \simeq 1088(\sim 33 \sigma)$.

The appearance of new sources within the ROI could in principle strongly influence the $\gamma$-ray spectrum and lightcurve of the investigated source. In order to investigate this we also searched for possible new $\gamma$-ray sources within a FOV of $20^{\circ}$. Our analysis of eight years of Fermi-LAT data does not show any evidence of additional transient sources beyond the 3FGL catalog.

We produced the $\gamma$-ray SED and light curves of M 87 through the unbinned and binned maximum likelihood fitting technique, respectively with gtlike to determine the flux and TS value for each time bin. Both energy and temporal bins with TS $<9$ (or $\sim 3 \sigma$ ) were set as upper limits throughout the paper. The effect of energy dispersion below $300 \mathrm{MeV}$ is accounted for in the analysis by enabling the energy dispersion correction.

\footnotetext{
1 http://fermi.gsfc.nasa.gov/ssc/data/analysis/ software

2 http://fermi.gsfc.nasa.gov/ssc/data/access/lat/ BackgroundModels.html
}

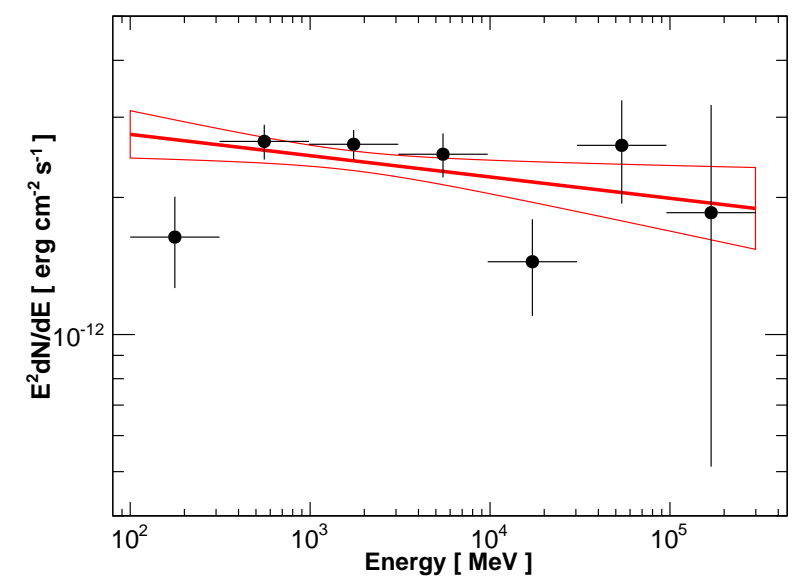

Fig. 1. SED of M 87 in the energy band of $100 \mathrm{MeV}-300 \mathrm{GeV}$ as extracted from the full (2008-2016) data set along with a power law fit. There are indications for an inflexion point around $\sim(5-30) \mathrm{GeV}$ with additional spectral hardening toward higher energies, which appears to suggest an additional emission component.

\section{High energy gamma-ray SED of M 87}

The HE emission of M 87 reveals hints of a deviation from a simple power law in the first 10 months of LAT data (Abdo et al. 2009) while the four-year (3FGL) update indicates a possible downturn at energies $\sim 10 \mathrm{GeV}$ (Acero et al. 2015). Our current analysis based on approximately eight years of LAT data provides additional indications for a deviation from a single power law and excess emission above $\gtrsim 10 \mathrm{GeV}$.

The SED for the full time and energy range (using 7 energy bins), is shown in Fig. 1 We performed a maximum likelihood analysis exploring different spectral forms for the whole energy range, namely single power-law (PL), broken power-law (BPL) and log-parabola (LP). The likelihood ratio test comparison yields $\mathrm{TS}=-2 \log \left(L_{\mathrm{PL}} / L_{\mathrm{BPL}}\right) \simeq 7.1$, thus preferring BPL over PL at a significance level of $\sim 2.66 \sigma$. The best fit indices are $\Gamma_{1}=1.79 \pm$ 0.15 and $\Gamma_{2}=2.18 \pm 0.06$ with the break at $E_{\mathrm{b}}=1.32 \pm 0.34 \mathrm{GeV}$. Here, $\Gamma_{2}>\Gamma_{1}$ due to the curvature below $1 \mathrm{GeV}$, as evident from the $E^{2} \mathrm{~d} N / \mathrm{d} E$ in Fig. 1. This is also suggested by the preference of $2.1 \sigma$ of LP over PL; both are nested, so that the preference can be readily evaluated applying Wilks' theorem.

To minimize the influence of the curvature seen at lower energies and to study the spectral extension toward higher energies, we also fit the SED points above $1 \mathrm{GeV}$. The results give a preference for a BPL over a PL at $2.4 \sigma$, with a break at $\sim 28 \pm 11 \mathrm{GeV}$, and indices $\Gamma_{1}=2.16 \pm 0.16$ and $\Gamma_{2}=1.89 \pm 0.29$, respectively (cf. Fig. 2). A similar outcome is obtained once $E_{\mathrm{b}}$ is fixed separately to truely satisfy Wilks' theorem. We note that this preference is comparable to the initial $(<3 \sigma)$ indications for spectral hardening at GeV energies in Cen A (Sahakyan et al. 2013); given additional and more sensitive data, this preference has now increased to $\sim 5 \sigma$ (Brown et al.2017). In the case of M 87, the precise position of the possible break cannot be easily improved given available data as sufficient statistics necessitate wider bins. Nevertheless, the indications of a preference for a inverted BPL over a PL model above $1 \mathrm{GeV}$ at $\gtrsim 2.5 \sigma$ suggests a non-trivial departure from the usual single component PL model. The outcome of an excess or inflexion above a few $\mathrm{GeV}$ critically depends upon the chosen bins at energies 5.5, 17.2 and 54.0 GeV in Fig. 1, each of which is, however, significant with TS values of 328, 70 and 56 respectively.

The above spectral analysis provides a hint for possible spectral inflexion at $\mathrm{GeV}$ energies. Such a situation might be expected if the source SED would actually be composed of more 


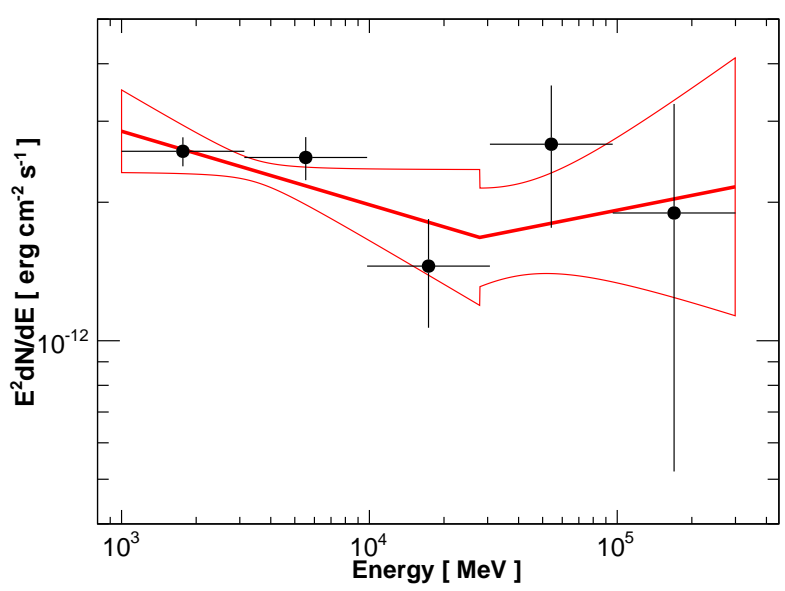

Fig. 2. SED of M 87 above $1 \mathrm{GeV}$ for the full (2008-2016) data set along with the preferred broken power law fit.

than one component. While not yet strong on its own, this possibility gains plausibility when viewed in the wider HE-VHE context (see Sect. 6). To experimentally improve the spectral characterization toward higher energies more sensitive data are needed, preferentially from lower threshold VHE observations by HESS, MAGIC and VERITAS or in time with CTA.

\section{Variability analysis}

Early HE studies find no evidence for significant variability during previous observations (Abdo et al. 2009; Aliu et al. 2012), though suggestions do exist in the 3FGL lightcurve (Acero et al. 2015). On the other hand, at VHE energies M 87 has shown at least three high flux states in 2005, 2008, and 2010 (e.g., Aharonian et al. 2006; Albert et al. 2008; Aliu et al. 2012), displaying extreme variability down to timescales of $\mathrm{O}(\sim 1$ day). Given the extended data set with longer observation window and relatively better statistics, we investigate anew for possible HE variability. Figure 3 shows the full lightcurve with data from 2008 to 2016 in the energy range $100 \mathrm{MeV}-300 \mathrm{GeV}$ using a binning of six months. Clear variations of a factor of approximately two over timescales of few months are apparent in the lightcurve. Fitting a constant yields $\chi^{2} / v=28.1 / 14$ with a probability $p<0.014$ for being constant $(\sim 2.5 \sigma)$. The six-month binning ensures an optimal choice, giving both sufficiently high statistics as well as a sufficiently high number of HE data points to investigate variations on timescales of several months. Given the weakness of the source, shorter-term variations are in general difficult to probe.

The considerations in Sect. 3 suggest a possible spectral inflexion somewhere between 5 and $30 \mathrm{GeV}$. Motivated by the spectral shape in Fig. 1, the lightcurve is split into two energy bands, (0.1-10) GeV and (10-300) GeV, to investigate variability above and below a potential break or inflexion point $E_{\mathrm{b}}$ :

(i) For the first band, between $0.1-10 \mathrm{GeV}$, we again employ a 6-month binning, leading to 15 bins as for the full band. The corresponding lightcurve shows variability on similar timescales as before (see Fig. 4). This is expected as the dominant statistical contribution to the full lightcurve comes from this part. Fitting a constant to this band results in $\chi^{2}=27.32$ for $14^{\circ}$ of freedom with $p$-value $\sim 0.018$. This confirms for the first time that the $\mathrm{HE}$ emission below $10 \mathrm{GeV}$ is variable at least on timescales of a few months.

(ii) Above $10 \mathrm{GeV}$, on the other hand, we find that the six-month binning yields several upper limits due to limited statis-

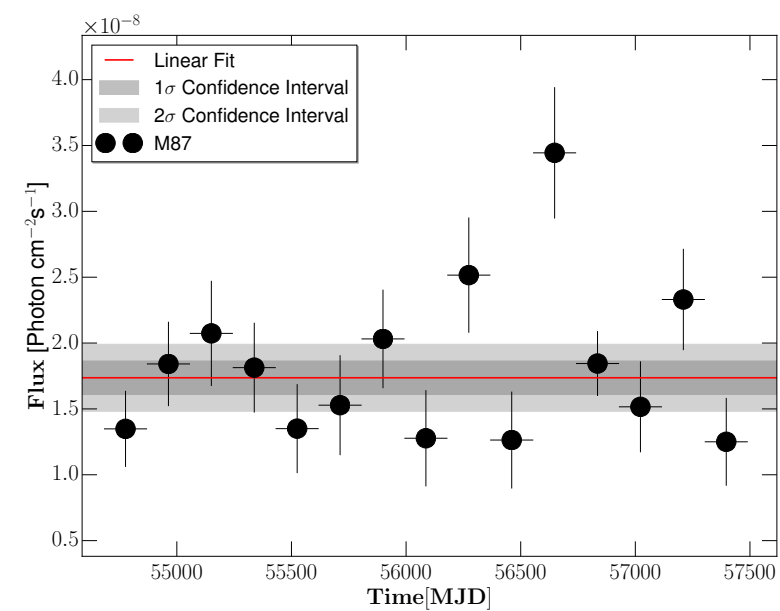

Fig. 3. $\gamma$-ray lightcurve of M 87 from 2008 to 2016 in the energy range $0.1-300 \mathrm{GeV}$. A six-month binning is employed. The red line gives a fit with a constant flux, while the shaded areas show the $1 \sigma$ and $2 \sigma$ confidence levels, respectively. There is clear evidence for variability. Error bars given are one-sigma error bars.

tics and hence we use a 24 month binning ensuring that there are no upper limits. A constant fit yields $\chi^{2}=4.33$ for 3 d.o.f. with a $p$-value $\sim 0.23$. Thus, variability in the high energy band $\gtrsim 10 \mathrm{GeV}$ is not statistically significant and would require higher statistics per bin and larger number of bins to be clearly ruled out or established. Similar to the SED, this deserves further investigation with lower threshold $\mathrm{TeV}$ observations. The evidence for variability below $10 \mathrm{GeV}$ seems consistent with, for example, expectations based on one-zone SSC modeling of its SED up to the GeV regime. Detection of differences in the variability behavior above and below $E_{\mathrm{b}}$ could in principle provide further evidence for multiple components.

\section{Changes in spectral state}

The spectral state shown in Fig. 1 is a long-term average SED of all the data from 2008 to 2016. This interval is known to encompass different source states at other energies, such as the major TeV flare recorded in 2010 (Abramowski et al. 2012; Aliu et al. 2012). The HE lightcurve analysis reported in Sect. 4 provided clear signs for a slow (monthly) variability below $E_{\mathrm{b}} \sim 10 \mathrm{GeV}$, yet nothing conclusive for the regime above $E_{\mathrm{b}}$. It seems interesting to explore whether an analysis of the spectrum over time allows further insights into the possible variability behavior of the two components. Inspection of the lightcurve below $E_{\mathrm{b}}$ reveals a rather high flux state in a single bin extending from September 2013 to March 2014. To study its impact, we split the whole dataset into this "high state" subset consisting of six months of data, and a subset called "regular state" consisting of the remaining data from 2008-2016. We note that the regular state in HE includes a strong short VHE flaring episode during April 5-15, 2010 (Aliu et al. 2012). Further subdivision of the current dataset into intermediate states analogous to the $\mathrm{TeV}$ states remains non-conclusive given that the HE statistics is limited and that the $\mathrm{GeV}$ and $\mathrm{TeV}$ observations of the high states are not simultaneous. A Bayesian blocks representation, that partitions the lightcurve into piecewise constant blocks by optimizing a fitness function (Scargle et al. 2013), also does not favor further states when the standard geometric prior is employed.

The respective SEDs are shown in Fig. 5. For the regular state an inflexion in the SED between $\sim(5-30) \mathrm{GeV}$ seems again 

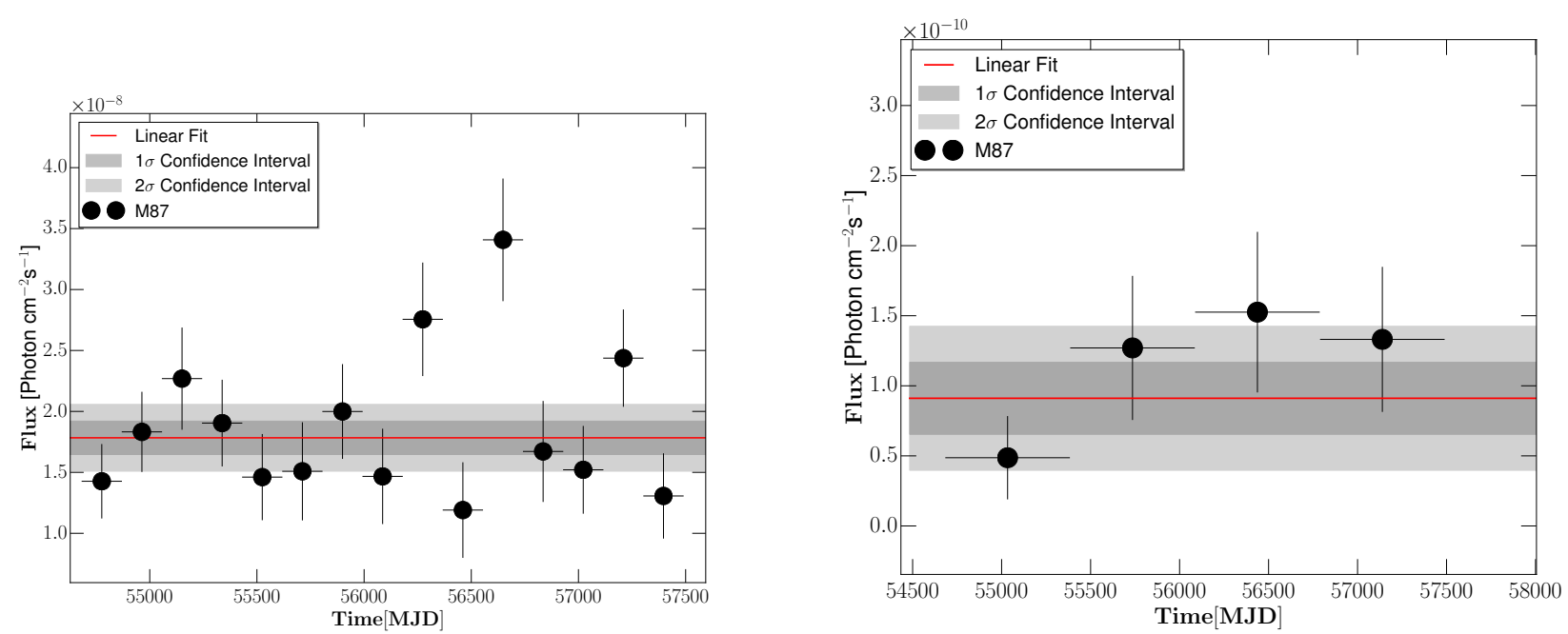

Fig. 4. Left panel: $\gamma$-ray lightcurve of M 87 from 2008 to 2016 in the energy range $100 \mathrm{MeV}-10 \mathrm{GeV}$. The binning here is six months. There are clear deviations from a constant flux level. Right panel: lightcurve in the energy band $10 \mathrm{GeV}-300 \mathrm{GeV}$ with a binsize of approximately two years. Here, statistics are not sufficiently large to establish variability. One-sigma error bars are shown.

indicated. On comparing the broken power-law model with the simple power-law model, we find a preference for the former at the level of $2.55 \sigma$ for the regular state, whereas there is no significant preference for BPL in the high state. The high state SED instead shows no remarkable drop but a rather smooth continuation (the inflexion being camouflaged) compatible with a single power law. The above analysis suggests, however, that this continuation may be caused by a rather smooth superposition of two physically different components. In fact, the observed spectral changes and the HE-VHE connection support the notion that the second component, which dominates the regular-state emission above $E_{\mathrm{b}}$ is variable and that its variability shapes the high-state SED. Though beyond the scope of the present paper, it would be interesting to explore possible spectral changes as a function of HE flux levels in more detail.

\section{Discussion and conclusions}

The analysis of the whole dataset provides evidence for monthtype variability and indications for a possible spectral turnover in the $\mathrm{GeV}$ regime $E_{\mathrm{b}} \gtrsim 10 \mathrm{GeV}$. The latter seems reminiscent of the spectral hardening seen in the nearest radio galaxy, Cen A (Sahakyan et al. 2013; Brown et al. 2017).

The current findings are most naturally interpreted as an additional physical component beyond the conventional SSC jet contribution for a misaligned AGN. Our variability analysis indicates that the component dominating the low energies $\left(E \leq E_{\mathrm{b}}\right)$ varies by a factor of approximately two on a timescale $\Delta t$ of at least a few months, which seems compatible with a jet-related origin. While the analysis did not ascertain variability of the second component, the observed spectral changes suggest that this component is variable on comparable timescales. This would disfavor steady or extended $\gamma$-ray production scenarios for its origin, such as inverse Compton scattering in its kiloparsec-scale jet (Hardcastle \& Croston 2011), dark matter annihilation (e.g., Baltz et al. 2000; Saxena et al. 2011; Lacroix et al. 2015) or cosmic-ray interactions in the interstellar medium (e.g., Pfrommer \& Enßlin 2003).

The current results instead rather favor a second component on scales of the inner jet and below, that is, on scales $d \lesssim 1300(\Delta t / 1$ month $) r_{g}$ for a jet half width to length ratio of 0.1 . Its true physical nature however, cannot yet be unam-

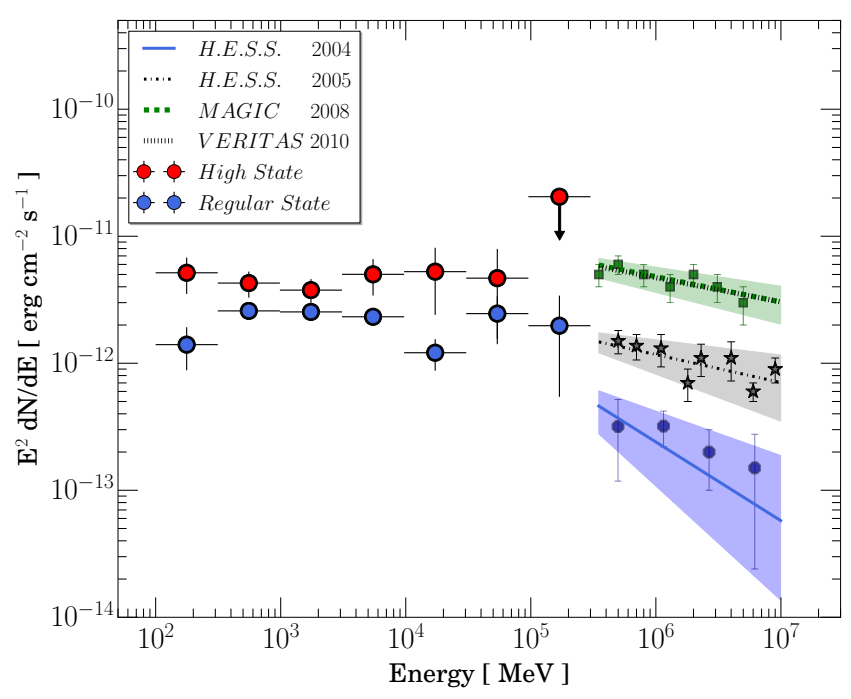

Fig. 5. $\gamma$-ray SED of M 87 for the regular and the high HE source state, respectively. The "high state" encompasses Fermi-LAT data from $09 / 2013$ to $03 / 2014$ and the "regular state" refers to the remaining data from 2008-2016. This regular state shows a break in the SED around $E \sim(5-30) \mathrm{GeV}$, as in Fig. 1, suggestive of an additional HE component. The break appears camouflaged in the high state by flaring above $\sim 10 \mathrm{GeV}$. Lines and shaded regions, including VHE data points, indicate published best fit power laws and confidence bands for previous VHE measurements (blue line: H.E.S.S. VHE low state in 2004, Aharonian et al. 2006; black dash-dotted: H.E.S.S. VHE high state in 2005, Aharonian et al. 2006; green dotted: MAGIC VHE high state in February 2008, Albert et al. 2008; black dotted: VERITAS VHE high state in April 2010, Aliu et al. 2012). Despite non-simultaneity, the observed HE spectral variability would now in principle allow matching the HE-VHE states.

biguously inferred and a variety of different interpretations appear possible. Proposals in the literature encompass scenarios wherein the gamma-ray emission in M 87 is attributed to leptonic or hadronic processes, or a combination thereof. The former range from inverse Compton scattering in a decelerating flow profile (Georganopoulos et al. 2005) or a spine-shear topology (Tavecchio \& Ghisellini 2008) via multiple SSC emission zones (Lenain et al. 2008) or various reconnection-driven events (e.g., 
Giannios et al. 2010) up to dynamic processes in its rotating black hole magnetosphere (e.g., Neronov \& Aharonian 2007; Rieger \& Aharonian 2008; Levinson \& Rieger 2011). Hadronic proposals, on the other hand, include $p \gamma$ and proton synchrotron processes (Reimer et al. 2004) or jet-star induced ppinteractions (e.g., Barkov et al. 2012), as well as complex lepto-hadronic realizations thereof (e.g., Reynoso et al. 2011; Fraija \& Marinelli 2016). Most of these scenarios introduce additional parameters and no longer aim at describing the overall low energy (submm)-VHE core SED by a single zone as attempted in earlier SSC models. They can thus potentially be modified to accommodate the current findings.

The connection of the HE and VHE regimes may deserve further investigations in this regard as it may eventually allow to substantiate general challenges associated with each of these scenarios (see Rieger \& Aharonian 2012, for a discussion). In the VHE monitoring campaign by MAGIC during the period 2012-2015 (Dec-July each year, with $\sim 40 \mathrm{~h}$ each year) no VHE flares were detected, although the VHE lightcurve above $300 \mathrm{GeV}$ seems to provide some hints for (day-scale) flickering in 2013 (Bangale et al. 2016). Some caution is needed, though, as the visibility at the MAGIC site (from Dec-July) constrains comparison, whereas the Fermi-LAT sensitivity limits the detectability of short flares above $\sim 10 \mathrm{GeV}$ (as might have been the case in April 2010). Nevertheless, if these findings hold up to closer scrutiny, it would suggest that (1) under normal circumstances, flux changes associated with the second component, do not necessarily imply strong changes at VHE $\gamma$-rays, in other words during a common HE increase its $\gamma$-ray SED peak tends to get shifted to lower energies. (2) On the other hand, during extraordinary VHE high states a reverse trend occurs, meaning the $\gamma$-ray peak of the second component gets shifted to higher energies. Clearly then, the cross-over regime between $\mathrm{HE}$ and VHE, that is, between space-based Fermi-LAT and groundbased Cherenkov arrays is particularly interesting.

The current Fermi-LAT analysis allows extraction of (significant) spectral points up to $\sim 100 \mathrm{GeV}$. At VHE energies, MAGIC reported a threshold of $300 \mathrm{GeV}$ for the monitoring noted above, while a detection down to $150 \mathrm{GeV}$ is known for the 2008 high state (Albert et al. 2008). With existing performance upgrades such as H.E.S.S. II (Abdalla et al. 2017) full energy overlap should thus soon be achievable.

In a minimalist approach, the fact that the present FermiLAT spectrum extends up to about $100 \mathrm{GeV}$ without indications for a strong cut-off and the case that current VHE thresholds reach down to about $200 \mathrm{GeV}$ leaves rather little room but to attribute both the HE emission above $E_{\mathrm{b}}$ and the VHE emission to anything other than the same physical component. While the limited sensitivity of Fermi-LAT for a weak source such as M 87 does not allow to probe deeper into the variability at high energies, ground-based Cherenkov instruments have found the VHE emission to be variable down to timescales of days (e.g., Aharonian et al. 2006; Albert et al. 2008; Acciari et al. 2009; Aliu et al. 2012). Given the scenario of the same component in both energy regimes, the day-scale variability seen at VHE imposes a general constraint on the overall nature, size $(r \lesssim$ $5\left[\Delta t_{\mathrm{VHE}} / 1\right.$ day $] r_{g}$ for negligible Doppler boosting) and location of this component $\left(d \lesssim 100 r_{g}\right.$ ). Unless further components are invoked, changes in the flux state of this component would then be responsible for the different states seen in the HE (above $E_{\mathrm{b}}$ ) and VHE domain as noted above. These considerations would then also suggest that the VHE emission during the quiescent state originates in a similar region as during the flare, further favoring inner-jet type scenarios (e.g., Acciari et al. 2009). Sug- gestions for a possible longterm evolution of the "quiescent" VHE state (Beilicke et al. 2012) appear to be consistent with this. The findings presented here provide some further illustration that nearby radio galaxies are carrying a prime potential to probe deeper into the physics and nature of AGN (e.g., Rieger 2017), motivating further VHE observations to truly disentangle the origin of the emission seen.

Acknowledgements. NC kindly acknowledges support by the AvH foundation and MPIK, and FMR by a DFG Heisenberg Fellowship (RI 1187/4-1). We thank Werner Hofmann and Luigi Tibaldo for insightful comments. We also thank the referee for the comments that were useful in improving the paper.

\section{References}

Abdalla, H., Abramowski, A., Aharonian, F., et al. 2017, A\&A, 600, A89

Abdo, A. A., Ackermann, M., Ajello, M., et al. 2009, ApJ, 707, 55

Abramowski, A., Acero, F., Aharonian, F., et al. 2012, ApJ, 746, 151

Acciari, V. A., Aliu, E., Arlen, T., et al. 2009, Science, 325, 444

Acero, F., Ackermann, M., Ajello, M., et al. 2015, ApJS, 218, 23

Aharonian, F., Akhperjanian, A., Beilicke, M., et al. 2003, A\&A, 403, L1

Aharonian, F., Akhperjanian, A. G., Bazer-Bachi, A. R., et al. 2006, Science, 314,1424

Albert, J., Aliu, E., Anderhub, H., et al. 2008, ApJ, 685, L23

Aliu, E., Arlen, T., Aune, T., et al. 2012, ApJ, 746, 141

Atwood, W. B., Abdo, A. A., Ackermann, M., et al. 2009, ApJ, 697, 1071

Atwood, W., Albert, A., Baldini, L., et al. 2013, 2012 Fermi Symposium Proceedings - eConf C121028, [arXiv:1303.3514]

Baltz, E. A., Briot, C., Salati, P., Taillet, R., \& Silk, J. 2000, Phys. Rev. D, 61, 023514

Bangale, P., Manganaro, M., Schultz, C., Colin, P., \& Mazin, D. 2016, PoS, ICRC2015, 759

Barkov, M. V., Bosch-Ramon, V., \& Aharonian, F. A. 2012, ApJ, 755, 170

Beilicke, M., Baring, M. G., Barthelmy, S., et al. 2012, AIP Conf. Ser., 1505, 586

Bird, S., Harris, W. E., Blakeslee, J. P., \& Flynn, C. 2010, A\&A, 524, A71

Brown, A. M., Böhm, C., Graham, J., et al. 2017, Phys. Rev. D, 95, 063018

Chiaberge, M., Capetti, A., \& Celotti, A. 2001, MNRAS, 324, L33

Doeleman, S. S., Fish, V. L., Schenck, D. E., et al. 2012, Science, 338, 355

Fraija, N., \& Marinelli, A. 2016, ApJ, 830, 81

Gebhardt, K., \& Thomas, J. 2009, ApJ, 700, 1690

Georganopoulos, M., Perlman, E. S., \& Kazanas, D. 2005, ApJ, 634, L33

Giannios, D., Uzdensky, D. A., \& Begelman, M. C. 2010, MNRAS, 402, 1649

Hada, K., Giroletti, M., Kino, M., et al. 2014, ApJ, 788, 165

Hada, K., Kino, M., Doi, A., et al. 2016, ApJ, 817, 131

Hardcastle, M. J., \& Croston, J. H. 2011, MNRAS, 415, 133

Kovalev, Y. Y., Lister, M. L., Homan, D. C., \& Kellermann, K. I. 2007, ApJ, 668, L27

Kuo, C. Y., Asada, K., Rao, R., et al. 2014, ApJ, 783, L33

Lacroix, T., Boehm, C., \& Silk, J. 2015, Phys. Rev. D, 92, 043510

Lenain, J.-P., Boisson, C., Sol, H., \& Katarzyński, K. 2008, A\&A, 478, 111

Levinson, A., \& Rieger, F. 2011, ApJ, 730, 123

Marconi, A., Axon, D. J., Macchetto, F. D., et al. 1997, MNRAS, 289, L21

Marshall, H. L., Miller, B. P., Davis, D. S., et al. 2002, ApJ, 564, 683

Mattox, J. R., Bertsch, D. L., Chiang, J., et al. 1996, ApJ, 461, 396

Mertens, F., Lobanov, A. P., Walker, R. C., \& Hardee, P. E. 2016, A\&A, 595, A54

Neronov, A., \& Aharonian, F. A. 2007, ApJ, 671, 85

Pfrommer, C., \& Enßlin, T. A. 2003, A\&A, 407, L73

Reimer, A., Protheroe, R. J., \& Donea, A.-C. 2004, A\&A, 419, 89

Reynolds, C. S., Di Matteo, T., Fabian, A. C., Hwang, U., \& Canizares, C. R. 1996, MNRAS, 283, L111

Reynoso, M. M., Medina, M. C., \& Romero, G. E. 2011, A\&A, 531, A30

Rieger, F. M. 2017, AIP Conf. Ser., 1792, 020008

Rieger, F. M., \& Aharonian, F. A. 2008, A\&A, 479, L5

Rieger, F. M., \& Aharonian, F. 2012, Mod. Phys. Lett. A, 27, 1230030

Sahakyan, N., Yang, R., Aharonian, F. A., \& Rieger, F. M. 2013, ApJ, 770, L6

Saxena, S., Summa, A., Elsässer, D., Rüger, M., \& Mannheim, K. 2011, Eur. Phys. J. C, 71, 1815

Scargle, J. D., Norris, J. P., Jackson, B., \& Chiang, J. 2013, ApJ, 764, 167

Tavecchio, F., \& Ghisellini, G. 2008, MNRAS, 385, L98

Walsh, J. L., Barth, A. J., Ho, L. C., \& Sarzi, M. 2013, ApJ, 770, 86 